\title{
PATTERNS OF RESPONSE AND DRUGS INVOLVED IN HYPERSENSITIVITY REACTIONS TO BETA-LACTAMS IN CHILDREN.
}

\author{
Isabel Torres-Rojas ${ }^{1}$, Diana Perez ${ }^{1}$, Maria Luisa Somoza-Alvarez ${ }^{1}$, Elisa Haroun Diaz ${ }^{1}$, \\ Ana María Prieto-Moreno Pfeifer ${ }^{1}$, Teodorikec Jimenez-Rodriguez ${ }^{2}$, Javier Fernandez ${ }^{3}$, \\ Miguel Blanca ${ }^{1}$, Gabriela Canto ${ }^{4}$, and Natalia Blanca-Lopez ${ }^{1}$ \\ ${ }^{1}$ Hospital Universitario Infanta Leonor \\ ${ }^{2}$ Alicante General University Hospital \\ ${ }^{3}$ Universidad Miguel Hernández \\ ${ }^{4}$ Infanta Leonor Hospital
}

March 21, 2021

\begin{abstract}
Background Beta-lactams generate different allergenic determinants that induce selective or cross-reactive drug hypersensitivity reactions (DHRs). We aimed to identify the drugs involved, the selectivity of the response, the mechanism, and the value of the different diagnostic tests for establishing a diagnosis in children evaluated for DHRs to beta-lactams. Methods Prospective study evaluating children aged under 16 years reporting DHRs to beta-lactams. Reactions were classified as immediate and nonimmediate reactions. The work-up included sIgE, skin testing and drug provocation tests (DPTs) for immediate reactions and patch testing and DPTs for nonimmediate ones. Results Of the 510 included children, 133 were evaluated for immediate reactions and confirmed in $8.3 \%$. Skin test/in vitro IgE contributed to diagnosing half of the cases. Selective reactions occurred with amoxicillin (63\%), followed by common penicillin determinants $(27 \%)$ and cephalosporins $(0.9 \%)$. Among nonimmediate reactions (11,4\% of the 377 children evaluated), most required DPTs, $52.7 \%$ of which were positive at $6-7$ days of drug challenge. Selective reactions were identified with amoxicillin (80\%), penicillin G (7.5\%), cephalosporins (7.5\%), and clavulanic acid (5\%). Urticaria and maculopapular exanthema were the most frequent entities. Conclusions There were few confirmed cases of either type of reaction. Skin testing proved less valuable in nonimmediate reactions, over half of which would also have been lost in a short DPT protocol. Selective responders to amoxicillin were more likely to have nonimmediate reactions, while clavulanic acid-selectivity was exclusive to the nonimmediate typology. Over half the cases with DPTs required 6-7 days of treatment for DHR confirmation.
\end{abstract}

Title : Patterns of response and drugs involved in hypersensitivity reactions to beta-lactams in children.

\section{Conflict of interest statement:}

The authors declare that there are no conflicts of interest.

Financial support:

This work was supported by grants from the ISCIII (PI20/00771), FEDER funds, and the national network ARADyAL (RD16/0006/0024; RD16/0006/0032).

Statement of Ethics: 
Ethical approval: The study was approved by our Institutional Ethics Committee.

Informed consent: a written informed consent was obtained from parents or guardians of all individual participants included in the study.

\section{Background}

Beta-lactams generate different allergenic determinants that induce selective or cross-reactive drug hypersensitivity reactions (DHRs). We aimed to identify the drugs involved, the selectivity of the response, the mechanism, and the value of the different diagnostic tests for establishing a diagnosis in children evaluated for DHRs to beta-lactams.

\section{Methods}

Prospective study evaluating children aged under 16 years reporting DHRs to beta-lactams. Reactions were classified as immediate and nonimmediate reactions. The work-up included sIgE, skin testing and drug provocation tests (DPTs) for immediate reactions and patch testing and DPTs for nonimmediate ones.

ResultsOf the 510 included children, 133 were evaluated for immediate reactions and confirmed in 8.3\%. Skin test/in vitro IgE contributed to diagnosing half of the cases. Selective reactions occurred with amoxicillin (63\%), followed by common penicillin determinants (27\%) and cephalosporins $(0.9 \%)$.

Among nonimmediate reactions (11,4\% of the 377 children evaluated), most required DPTs, $52.7 \%$ of which were positive at 6-7 days of drug challenge. Selective reactions were identified with amoxicillin (80\%), penicillin G (7.5\%), cephalosporins (7.5\%), and clavulanic acid $(5 \%)$. Urticaria and maculopapular exanthema were the most frequent entities.

\section{Conclusions}

There were few confirmed cases of either type of reaction. Skin testing proved less valuable in nonimmediate reactions, over half of which would also have been lost in a short DPT protocol. Selective responders to amoxicillin were more likely to have nonimmediate reactions, while clavulanic acid-selectivity was exclusive to the nonimmediate typology. Over half the cases with DPTs required 6-7 days of treatment for DHR confirmation.

Key words: hypersensitivity, betalactams, immediate reaction, nonimmediate reactions, children.

\section{Key Message}

Beta-lactams are the first-line antibiotic to control many bacterial infections. Traditionally, the most frequently prescribed antibiotic in children has been amoxicillin, which has been increasingly combined with clavulanic acid. An accurate diagnosis is essential for avoiding the prescription of alternative antibiotics which may be less effective, more toxic. Betalactams drug hypersensitivity reactions are classified as immediate and nonimmediate, being the last one the most frequent in children. The aim of our study was to identify the drugs involved, the selectivity of the response, the mechanism, and the value of the different diagnostic tests for establishing a diagnosis in children evaluated for DHRs to BLs. After the allergollogical study we conclude that only few cases were confirmed of either type of reaction. Skin testing proved less valuable in nonimmediate reactions, over half of which would also have been lost in a short DPT protocol. Selective responders to amoxicillin were more likely to have nonimmediate reactions, while clavulanic acidselectivity was exclusive to the nonimmediate typology. Over half the cases with DPTs required 6-7 days of treatment for DHR confirmation.

\section{Introduction}

Beta-lactams (BLs) are the first-line antibiotic to control many bacterial infections ${ }^{1}$. Traditionally, the most frequently prescribed antibiotic in children has been amoxicillin (AX), which has been increasingly combined with clavulanic acid (CLAV); in recent years, this formulation has become the most dominant ${ }^{1-2}$. BLs are also the most common inducers of drug hypersensitivity reactions (DHR) in children. Since systematic 
reporting of side-chain-specific reactions to AX were first published in the late 1980s, diagnosis has required more determinants for evaluating $\mathrm{DHRs}^{3}$. In many countries, penicillin G (PG)(benzylpenicillin) is no longer the major determinant, and classical penicillin determinants yield low sensitivity, making drug provocation tests (DPTs) necessary for diagnosis ${ }^{4}$.

The prevalence of DHR in children ranges from $2.5 \%$ to $10.2 \%^{5}$. More than $10 \%$ of children develop skin rashes over the course of an antibiotic treatment for a viral infection. Allergological evaluation confirms that only a few cases are confirmed allergic ${ }^{5,6}$, while most skin exanthemas are due to underlying viral infections or to interactions between drugs and infectious agents ${ }^{5,7,8}$.

An accurate diagnosis is essential for avoiding the prescription of alternative antibiotics which may be less effective, more toxic, and larger contributors to bacterial resistance. Significant differences exist between European centers in terms of diagnosing BL hypersensitivity, particularly in children ${ }^{7,9}$. Although consensus protocols have helped to ensure patient safety and accurate diagnosis, these must be adapted to betweencountry variations in both patients' response to BLs and health system capacities for diagnosis.

BLs DHRs are classified as immediate (IR) and nonimmediate reactions (NIR) ${ }^{10}$. IRs usually appear within $1 \mathrm{~h}$ after drug administration and include urticaria, angioedema, rhinitis, bronchospasm, and anaphylaxis ${ }^{11}$. NIRs, although assumed to occur 24-48 h after drug intake ${ }^{5}$, can actually occur anytime from $1 \mathrm{~h}$ after taking the medication ${ }^{12}$. Clinical presentation ranges from mild reactions, such as nonimmediate urticaria (NIU) and maculopapular exanthema (MPE), to more severe reactions like acute generalized exanthematous pustulosis, drug-induced hypersensitivity syndrome, Stevens-Johnson syndrome, and toxic epidermal necrolysis (13).

The diagnostic approach varies depending on whether the reaction is immediate (IgE mediated) or nonimmediate ( $\mathrm{T}$ cell effector response), as well as the severity, symptoms elicited, and patient risk factors. Although they have low sensitivity, skin and SIgE testing may have a role in $\mathrm{IR}^{12,14}$. Several studies support direct DPT without skin testing (ST), especially in children with mild NIR ${ }^{7,15-17}$. However, if the results are positive, avoidance of the BL involved or all the drug group is controversial because reactions can be side-chain-specific to AX, cephalosporins, or another BLs, meaning that a different class of BL could still be administered $^{3,18}$.

The aim of our study was to identify the drugs involved, the selectivity of the response, the mechanism, and the value of the different diagnostic tests for establishing a diagnosis in children evaluated for DHRs to BLs.

\section{Material and methods}

\section{Patients}

This 10-year prospective study (2010-2019) included children aged under 16 years who were referred to our allergy unit for an evaluation of BL DHR.Reactions were classified as IR or NIR according to established criteria $^{5,10}$. For IRs, ST (prick tests and intradermal tests) were carried out as recommended, with some modifications $^{19}$. For NIRs, we also followed the pediatric adaptation ${ }^{5,8,21}$ of the 2004 general recommendations made by the European Network for Drug Allergy (ENDA) and the European Academy of Allergy and Clinical Immunology (EAACI) ${ }^{20}$, that is, ST (delayed intradermal tests and patch tests) followed by DPT when appropriate.

\section{Evaluation of IR}

Before ST, SIgE testing was carried out by fluoroimmunoassay (CAP system, Thermofisher Diagnosis, Uppsala, Sweden R). Results of $0.35 \mathrm{kUA} / 1$ or more were considered positive ${ }^{11}$.

The determinants used for ST were penicilloyl-polylysine (PPL) $\left(5 \times 10^{-5} \mathrm{~mol} / \mathrm{l}\right)$, minor determinant mixture $\left(2 \times 10^{-2} \mathrm{~mol} / \mathrm{l}\right)$, and $\mathrm{PG}\left(10^{4} \mathrm{UI} / \mathrm{ml}\right)$. If negative, we proceeded to the graded administration of penicillin $\mathrm{V}$ until achieving the therapeutic dose ${ }^{5,19}$. If tolerated well, we evaluated the response to AX if this drug was involved alone or in combination with CLAV; if tolerance was still good, we proceeded to ST with CLAV to test if this was the culprit, and then finally to a DPT with AX-CLAV. If a cephalosporin was involved, we 
tested first with common determinants of PG, and if negative, we proceeded to cephalosporin (first ST and if negative, DPT when indicated).

DPT was performed in a single-blind, placebo-controlled way as described elsewhere ${ }^{18}$, under strict hospital surveillance with emergency room facilities. We gave escalating doses of the drug at intervals of $30 \mathrm{~min}$ to 60 min until reaching the weight-adjusted therapeutic dose ${ }^{5}$. BLs were administered orally at the following doses: $5 \mathrm{mg}, 25 \mathrm{mg}, 50 \mathrm{mg}, 75 \mathrm{mg}, 100 \mathrm{mg}$, and $250 \mathrm{mg}$, with a final observation period of at least $1 \mathrm{~h}$ after the last dose administered.

Evaluation of NIR

Patch testing was performed on the patient's back, as described elsewhere ${ }^{22}$. Drugs were mixed in petrolatum $50 \% \mathrm{w} / \mathrm{w}$ at a final concentration of $20 \mathrm{mg}$. Readings were done at $48 \mathrm{~h}$ and $72 \mathrm{~h}$ after patch application, and if negative, patients were recommended to monitor the patch site to detect any eventual reaction. Due to the very low sensitivity of the test and the lack of systemic effects in cases with mild reactions ${ }^{5-10}$, in most instances all BLs were tested on the same day. In case of negative results, we proceeded to DPT as described ${ }^{5}$. DPT was considered positive if any objective symptoms indicative of an allergic reaction appeared during the treatment or within $48 \mathrm{~h}$ after the last dose $^{5}$.

Statistical analysis

A descriptive analysis of clinical characteristics was undertaken, and mean age, age range, gender distribution, and mean time between reaction and workup were calculated. The chi-square test was used to compare qualitative variables between patients with IR versus NIR. P values of less than 0.05 were considered significant. Mean age was compared using the Mann-Whitney U test.

\section{Results}

A total of 510 children (229 [45\%] girls; median age 4.3 years [range 0.5 to 16]) with a clinical history indicative of BL DHR were evaluated. Based on clinical history, AX was involved in $349(68.5 \%)$ cases, AX-CLAV in 123 (24.1\%), penicillin in 23 (4.5\%), and different cephalosporins in 15 (2.9\%).

After the allergological workup, 54 cases $(10.6 \%)$ were finally confirmed as allergic. The median time between the reaction and the allergological study was 30 days (range 1 to 1095). Of the 54 allergic patients, 30 (55.5\%) were girls, with an age range of 0.5 to 16 years (mean 6.2 ), and $42.6 \%$ were atopic. AX produced reactions in $72 \%$ of the positive cases, AX-CLAV acid in $20.4 \%$, and cephalosporins in $7.6 \%$. When comparing cases finally diagnosed as allergic versus non-allergic, we did not observe differences in gender or the BL involved, but we found differences in the mean age $(\mathrm{p}<0.001)$, which was higher in cases confirmed as allergic.

Among positive cases, $20.4 \%$ were classified as IRs and the $79.6 \%$ as NIRs. Figure 1 shows the allergological algorithm with the evaluation of all cases.

Immediate reactions

Diagnosis of IR was established in 11 of the 133 cases evaluated (8.3\%), nearly three-quarters $(72.7 \%)$ of whom were girls. Reactions were induced by AX (72.7\%), AX-CLAV (18.2\%), and cephalosporins (9.1\%).

$\mathrm{SIgE}$ was detected in two cases. One patient presented values of $\operatorname{sgE}$ to $\mathrm{AX}$ of $0.37 \mathrm{kU} / \mathrm{l}$; to ampicillin, $0.37 \mathrm{kU} / \mathrm{l}$; to $\mathrm{PG}, 0.36 \mathrm{kU} / \mathrm{l}$; and to $\mathrm{PV}, 0.35 \mathrm{kU} / \mathrm{l}$. Another showed $\operatorname{sIgE}$ to $\mathrm{AX}$ of $0.52 \mathrm{kU} / \mathrm{l}$; to ampicillin, $0.49 \mathrm{kU} / \mathrm{l}$; to $\mathrm{PG}, 0.37 \mathrm{kU} / \mathrm{l}$; and to $\mathrm{PV}, 0.35 \mathrm{kU} / \mathrm{l}$.

Different ST were positive in 4 out of 131 children, all to AX: by prick test at $20 \mathrm{mg} / \mathrm{ml}(\mathrm{n}=1)$, intradermal test at $2 \mathrm{mg} / \mathrm{ml}(\mathrm{n}=1)$, and intradermal test at $20 \mathrm{mg} / \mathrm{ml}(\mathrm{n}=2)$.

DPTs were performed in the remaining 127 patients, yielding positive results in 5 (Table 1), in all cases within 45 minutes or less. Three cases were positive to AX but showed good tolerance to PG and PV; one case was positive to PV; and one to cefaclor. Most of the reactions observed during the DPT were mild (urticaria in 1 case and angioedema in 3 cases), with only one child developing skin involvement plus 
bronchospasm and good recovery after treatment. The remaining 122 cases presented good tolerance to AX or another culprit BL until reaching therapeutic doses.

Is summary, of the total $8.3 \%$ positives cases within this group SIgE contributed to the diagnosis in the $1.5 \%$, ST with all the determinants in the $3 \%$ and DPT in the $3.7 \%$. Selective reactions occurred with AX $(63 \%)$, followed by common penicillin determinants $(27 \%)$ and cephalosporins $(0.9 \%)$.

Nonimmediate reactions

Of 377 patients evaluated for NIRs, diagnoses were confirmed in 43 (11.4\%). Half (51\%) were girls, and $39.5 \%$ atopic. The BLs involved were AX (72\%), AX-CLAV (21\%) and cephalosporins (7\%).

Severe reactions were reported in three cases (one acute generalized exanthematous pustulosis, one exudative erythema multiforme, and one drug-induced hypersensitivity syndrome). Patients were diagnosed based on their clinical history; neither ST nor DPT were carried out because of the risks.

Except for the three patients with severe reactions, patch testing was carried out in all other children evaluated $(\mathrm{n}=374)$. Two were positive to $\mathrm{AX}$, one to $\mathrm{PG}$, and one to cefotaxime.

A controlled DPT was performed in 370 children, yielding positive results in 36 cases (9.7\%) (Table 2). Thirty cases were positive to AX, all with good tolerance to PV; two were positive to CLAV, with good tolerance to PV and AX; two were positive to PV; one to cefaclor, and one to cefixime. These latter two cases both showed good tolerance to PV and to AX.

Clinical entities induced by DPT included NIU in 21 (58.3\%) cases (plus angioedema in 5), and MPE in 15 ( $41.6 \%$ )(See table 2). In all cases a full therapeutic dose was required to elicit a response. However, when we analyzed the interval from drug administration to reaction, there was a clear divergence: $30 \%$ of the positive cases presented a reaction within $24 \mathrm{~h}$, while $52.7 \%$ responded only at day 6 or 7 (Figure 2). Less than $10 \%$ of positives started showing symptoms between days 2 and 5 .

Is summary, of the total $11.4 \%$ positives cases within this group ST contributed to the diagnosis in the $1.1 \%$ and DPT in the 9.5\%. Selective reactions were identified with AX (80\%), PG (7.5\%), cephalosporins (7.5\%), and CLAV (5\%). NIU and MPE were the most frequent entities.

\section{Discussion}

This study included a large cohort of children referred to our center for BL allergy evaluation. We followed the classification of IR versus NIR, as initially reported by Terrados et al. ${ }^{10}$ and used extensively in the literature $8,16,18,21-25$.

Of the total patients evaluated, $10.6 \%$ were confirmed as allergic, most of whom were diagnosed by DPT. This proportion is lower than that reported by Ponvert et al. ${ }^{21}$, but consistent with other studies ${ }^{8,16,18,24}$.

Regarding the drugs involved, AX was implicated in $68.5 \%$ of the cases and AX-CLAV in $24 \%$, very similar to results reported elsewhere ${ }^{16}$. Penicillin and cephalosporins accounted for $4.5 \%$ and $3 \%$ of the positive cases, respectively. Of interest is the important role of AX-CLAV, coherent with the high pattern of prescription $^{1,2}$. The reported involvement of this formulation in DHR has changed from $12 \%^{8}$ to over $70 \%$ in recent years ${ }^{15,22,23}$. Considered less immunogenic than other $\mathrm{BLs}^{26}$, its contribution to both IR and NIR in adults has been reported ${ }^{27}$.

The proportion of confirmed IRs was $8.3 \%$, with positive ST or SIgE contributing in the $5.4 \%$. These values are lower than the $17 \%$ reported by Ibañez et al. ${ }^{16}$ but higher than the results observed by Mill et al. ${ }^{28}$ One study even reported a proportion of $86 \%$ positive $\mathrm{ST}^{21}$, but these data have not been supported by other studies ${ }^{8,16,23}$.

Regarding the different BLs, $63 \%$ of cases were selective reactors to AX, with no contribution from CLAV. This result contrasts with other studies published in adults, where $22 \%$ of participants were selective responders to $\mathrm{CLAV}^{27}$. 
Although AX was involved in $92.5 \%$ of the cases initially evaluated, $27 \%$ of the confirmed responders belonged to the common group of penicillin reactor. The contribution of cephalosporins was less than $1 \%$.

Symptoms suggestive of a NIR were reported in 377 cases, nearly $80 \%$ of the cases evaluated. Three cases with a severe reaction were diagnosed based on the clinical history. ST were positive in $1 \%$ of cases (2 cases to AX, 1 to PG and 1 to a cefotaxime). CLAV did not induce any positive ST. DPT was needed for confirmation in 36 of the 43 cases (83\%) finally classified as positive. In total, considering all cases diagnosed based on clinical history, ST or DPT, the final proportion of positive NIRs was $11.4 \%$. In our study, $52 \%$ of positive DPTs induced urticaria, with or without angioedema, while this reaction occurred in $72 \%$ of positive cases in the study by Ibañez et al. ${ }^{16}$ and $64 \%$ of cases included in Mori et al.'s report ${ }^{25}$. Data on the frequency of MPE versus NIU are variable in the literature. In large series like ours, the proportion of MPE reactions ranges anywhere from $18 \%$ to $80 \%^{15,18,22}$ which is in line with the $41.6 \%$ we observed in our study.

The interval between drug administration and symptoms onset was under an hour in IR, similar to what occurs in adults, although the dose required for eliciting a response was higher in children after correcting it for body weight ${ }^{29}$.

In NIRs, the analysis of the time required for inducing a positive DPT showed two response patterns. Up to a third of the cases were diagnosed on the first day, but over half the diagnoses required 6 or 7 days, which suggests that a 5-day protocol, as reported by some groups ${ }^{13,17,24}$, will miss an important number of positive cases. Other studies have also supported periods longer than 5 days ${ }^{26,27}$. In order to reconcile results from the different groups, responders with NIRs may fall into two broad groups, those responding early and those with a later response. This time interval is independent of the clinical entity induced (MPE or NIU), as shown in our work.

In NIRs, the role of selective responders to AX seems more relevant than in IRs, since $83 \%$ of cases with NIRs were selective in contrast with $63 \%$ of cases in IR. These data are similar to those reported in adults ${ }^{10}$.

One limitation of this study, as in other similar ones, is the lack of comparison between children showing a reaction at 2-3 days versus 5 days or longer. The low prevalence of allergy to BLs in children complicates this analysis. Also, we used patch testing, as done by other authors ${ }^{30,31}$ although there are studies suggesting that intradermal testing is more sensitive ${ }^{8,26}$.

Summarizing, after prospectively evaluating a large series of children with DHRs to BLs, we confirmed the diagnosis in $8.3 \%$ of the children assessed for IRs and 11,4\% of those assessed for NIRs. Overall, NIRs were more frequent and had a higher proportion of selective responders. Moreover, reactions to CLAV were exclusively NIRs.

\section{References}

1. Rossignoli A, Clavenna A, Bonati M. Antibiotic prescription and prevalence rate in the outpatient paediatric population: analysis of surveys published during 2000-2005. European journal of clinical pharmacology. 2007;63(12):1099-106.

2. Blanca M, Mayorga C, Torres MJ, Warrington R, Romano A, Demoly P, et al. Side-chain-specific reactions to betalactams: 14 years later. Clin Exp Allergy. 2002;32(2):192-7.

3. Ceelie I, van der Starre C, Tibboel D, Stol K, Koren G, de Wildt SN. Evaluation of drug formularies for pediatric intensive care. Pediatric critical care medicine : a journal of the Society of Critical Care Medicine and the World Federation of Pediatric Intensive and Critical Care Societies. 2011;12(1):e14-9.

4. Blumenthal KG, Li Y, Banerji A, Yun BJ, Long AA, Walensky RP. The Cost of Penicillin Allergy Evaluation. J Allergy Clin Immunol Pract. 2018;6(3):1019-27.e2.

5. Gomes ER, Brockow K, Kuyucu S, Saretta F, Mori F, Blanca-Lopez N, et al. Drug hypersensitivity in children: report from the pediatric task force of the EAACI Drug Allergy Interest Group. Allergy. 2016;71(2):149-61. 
6. Caubet JC, Frossard C, Fellay B, Eigenmann PA. Skin tests and in vitro allergy tests have a poor diagnostic value for benign skin rashes due to $\beta$-lactams in children. Pediatric allergy and immunology : official publication of the European Society of Pediatric Allergy and Immunology. 2015;26(1):80-2.

7. Tonson la Tour A, Michelet M, Eigenmann PA, Caubet JC. Natural History of Benign Nonimmediate Allergy to Beta-Lactams in Children: A Prospective Study in Retreated Patients After a Positive and a Negative Provocation Test. J Allergy Clin Immunol Pract. 2018;6(4):1321-6.

8. Atanaskovic-Markovic M, Gaeta F, Medjo B, Gavrovic-Jankulovic M, Cirkovic Velickovic T, Tmusic V, et al. Non-immediate hypersensitivity reactions to beta-lactam antibiotics in children - our 10-year experience in allergy work-up. Pediatric allergy and immunology : official publication of the European Society of Pediatric Allergy and Immunology. 2016;27(5):533-8.

9. Torres MJ, Celik GE, Whitaker P, Atanaskovic-Markovic M, Barbaud A, Bircher A, et al. A EAACI drug allergy interest group survey on how European allergy specialists deal with $\beta$-lactam allergy. Allergy. 2019;74(6):1052-62.

10. Terrados S, Blanca M, Garcia J, Vega J, Torres MJ, Carmona MJ, et al. Nonimmediate reactions to betalactams: prevalence and role of the different penicillins. Allergy. 1995;50(7):563-7.

11. Blanca-Lopez N, Jimenez-Rodriguez TW, Somoza ML, Gomez E, Al-Ahmad M, Perez-Sala D, et al. Allergic reactions to penicillins and cephalosporins: diagnosis, assessment of cross-reactivity and management. Expert review of clinical immunology. 2019;15(7):707-21.

12. Blanca M, Torres MJ, Mayorga C, Perez-Inestrosa E, Suau R, Montañez MI, et al. Immediate allergic reactions to betalactams: facts and controversies. Current opinion in allergy and clinical immunology. 2004;4(4):261-6.

13. Brockow K, Ardern-Jones MR, Mockenhaupt M, Aberer W, Barbaud A, Caubet JC, et al. EAACI position paper on how to classify cutaneous manifestations of drug hypersensitivity. Allergy. 2019;74(1):14-27.

14. Fontaine C, Mayorga C, Bousquet PJ, Arnoux B, Torres MJ, Blanca M, et al. Relevance of the determination of serum-specific IgE antibodies in the diagnosis of immediate beta-lactam allergy. Allergy. $2007 ; 62(1): 47-52$.

15. Vezir E, Dibek Misirlioglu E, Civelek E, Capanoglu M, Guvenir H, Ginis T, et al. Direct oral provocation tests in non-immediate mild cutaneous reactions related to beta-lactam antibiotics. Pediatric allergy and immunology : official publication of the European Society of Pediatric Allergy and Immunology. 2016;27(1):50-4.

16. Ibáñez MD, Rodríguez Del Río P, Lasa EM, Joral A, Ruiz-Hornillos J, Muñoz C, et al. Prospective assessment of diagnostic tests for pediatric penicillin allergy: From clinical history to challenge tests. Annals of allergy, asthma \& immunology : official publication of the American College of Allergy, Asthma, \& Immunology. 2018;121(2):235-44.e3.

17. Caubet JC, Kaiser L, Lemaître B, Fellay B, Gervaix A, Eigenmann PA. The role of penicillin in benign skin rashes in childhood: a prospective study based on drug rechallenge. J Allergy Clin Immunol. 2011;127(1):21822 .

18. Zambonino MA, Corzo JL, Muñoz C, Requena G, Ariza A, Mayorga C, et al. Diagnostic evaluation of hypersensitivity reactions to beta-lactam antibiotics in a large population of children. Pediatric allergy and immunology : official publication of the European Society of Pediatric Allergy and Immunology. 2014;25(1):80-7.

19. Torres MJ, Blanca M, Fernandez J, Romano A, Weck A, Aberer W, et al. Diagnosis of immediate allergic reactions to beta-lactam antibiotics. Allergy. 2003;58(10):961-72.

20. Romano A, Blanca M, Torres MJ, Bircher A, Aberer W, Brockow K, et al. Diagnosis of nonimmediate reactions to beta-lactam antibiotics. Allergy. 2004;59(11):1153-60. 
21. Ponvert C, Perrin Y, Bados-Albiero A, Le Bourgeois M, Karila C, Delacourt C, et al. Allergy to betalactam antibiotics in children: results of a 20-year study based on clinical history, skin and challenge tests. Pediatric allergy and immunology : official publication of the European Society of Pediatric Allergy and Immunology. 2011;22(4):411-8.

22. Kulhas Celik I, Guvenir H, Hurmuzlu S, Toyran M, Civelek E, Kocabas CN, et al. The negative predictive value of 5-day drug provocation test in nonimmediate beta-lactam allergy in children. Annals of allergy, asthma \& immunology : official publication of the American College of Allergy, Asthma, \& Immunology. 2020;124(5):494-9.

23. Piccorossi A, Liccioli G, Barni S, Sarti L, Giovannini M, Verrotti A, et al. Epidemiology and drug allergy results in children investigated in allergy unit of a tertiary-care paediatric hospital setting. Italian journal of pediatrics. 2020;46(1):5.

24. Lezmi G, Alrowaishdi F, Bados-Albiero A, Scheinmann P, de Blic J, Ponvert C. Non-immediate-reading skin tests and prolonged challenges in non-immediate hypersensitivity to beta-lactams in children. Pediatric allergy and immunology : official publication of the European Society of Pediatric Allergy and Immunology. $2018 ; 29(1): 84-9$.

25. Mori F, Cianferoni A, Barni S, Pucci N, Rossi ME, Novembre E. Amoxicillin allergy in children: fiveday drug provocation test in the diagnosis of nonimmediate reactions. J Allergy Clin Immunol Pract. 2015;3(3):375-80.e1.

26. Edwards RG, Dewdney JM, Dobrzanski RJ, Lee D. Immunogenicity and allergenicity studies on two beta-lactam structures, a clavam, clavulanic acid, and a carbapenem: structure-activity relationships. Int Arch Allergy Appl Immunol. 1988;85(2):184-9.

27. Blanca-Lopez N, Perez-Alzate D, Ruano F, Garcimartin M, de la Torre V, Mayorga C, et al. Selective immediate responders to amoxicillin and clavulanic acid tolerate penicillin derivative administration after confirming the diagnosis. Allergy. 2015;70(8):1013-9.

28. Mill C, Primeau MN, Medoff E, Lejtenyi C, O'Keefe A, Netchiporouk E, et al. Assessing the Diagnostic Properties of a Graded Oral Provocation Challenge for the Diagnosis of Immediate and Nonimmediate Reactions to Amoxicillin in Children. JAMA pediatrics. 2016;170(6):e160033.

29. Torres MJ, Mayorga C, Leyva L, Guzman AE, Cornejo-García JA, Juarez C, et al. Controlled administration of penicillin to patients with a positive history but negative skin and specific serum IgE tests. Clin Exp Allergy. 2002;32(2):270-6.

30. Blanca-López N, Zapatero L, Alonso E, Torres MJ, Fuentes V, Martínez-Molero MI, et al. Skin testing and drug provocation in the diagnosis of nonimmediate reactions to aminopenicillins in children. Allergy. 2009;64(2):229-33.

31. Vila L, Garcia V, Martinez Azcona O, Pineiro L, Meijide A, Balboa V. Mild to moderate hypersensitivity reactions to beta-lactams in children: a single-centre retrospective review. BMJ paediatrics open. 2019;3(1):e000435.

Table 1. Clinical characteristics of cases with confirmed IR by DPT

\begin{tabular}{lllll}
\hline Patient & Age (years) & Sex & Clinical Entity & Clinical characteristics (symptoms induced, dose, time from \\
\hline 1 & 7 & F & ANA & Cough and wheezing, with facial erythema and $20 \% \mathrm{FEV}_{1}$ decrease 10 \\
2 & 3 & F & URT & Facial erythema with eyelid angioedema and wheals on neck, 20 min a \\
3 & 6 & F & URT & Pruritus on face, ears and back, followed by generalized wheals and lin \\
4 & 1 & F & URT & Wheals in chest and face with pruritus 45 min after a cumulative dose \\
5 & 3 & F & AE & Eyelid angioedema with pruritus 45 min after a cumulative dose of 30 \\
\hline
\end{tabular}


F: female; M: male; ANA: anaphylaxis; URT: urticaria; AE: angioedema; AX: amoxicillin; PV: penicillin V.

Table 2. Clinical characteristics of cases with confirmed NIR by DPT

\begin{tabular}{|c|c|c|c|c|}
\hline Patient & Age (years) & Gender & Clinical entity & Clinical characteristics (symptoms induced, dose, time from DPT to re \\
\hline 1 & 2 & $\mathrm{~F}$ & MPE & Generalized MPE $6 \mathrm{~h}$ after taking $150 \mathrm{mg}$ AX ( $1^{\text {st }}$ day $)$. Good tolera \\
\hline 2 & 4 & $\mathrm{~F}$ & MPE & MPE on chest and legs with facial and retroarticular erythema and pr \\
\hline 3 & 15 & $\mathrm{~F}$ & MPE & Facial erythema and pruritus followed by MPE on chest $3 \mathrm{~h}$ after taki \\
\hline 4 & 10 & M & MPE & MPE on chest and abdomen after taking $250 \mathrm{mg}$ of AX every $8 \mathrm{~h}\left(2^{\text {nd }}\right.$ \\
\hline 5 & 5 & M & MPE & Isolated wheals on back after taking $250 \mathrm{mg}$ of AX every $8 \mathrm{~h}$ ( $6^{\text {th }}$ day). \\
\hline 6 & 1 & M & MPE & MPE on trunk, abdomen, and legs accompanied with pruritus $4 \mathrm{~h}$ after \\
\hline 7 & 9 & $\mathrm{~F}$ & MPE & MPE on abdomen, legs, and back accompanied with pruritus $8 \mathrm{~h}$ after \\
\hline 8 & 12 & $\mathrm{~F}$ & MPE & Pruritus and generalized MPE after taking $500 \mathrm{mg}$ of AX every $8 \mathrm{~h}(4$ \\
\hline 9 & 6 & M & MPE & MPE on chest and neck with pruritus after taking $250 \mathrm{mg}$ of AX every \\
\hline 10 & 11 & M & MPE & Wheals on face, abdomen, shoulders and legs with pruritus $8 \mathrm{~h}$ after tal \\
\hline 11 & 1 & M & MPE & Pruritus and MPE on abdomen and chest after taking $125 \mathrm{mg}$ of $\mathrm{AX}$ er \\
\hline 12 & 1.5 & $\mathrm{~F}$ & MPE & MPE on neck, back, arms and legs $1.5 \mathrm{~h}$ after taking $200 \mathrm{mg}$ of PV ( $1^{\text {st }}$ \\
\hline 13 & 15 & $\mathrm{~F}$ & MPE & MPE on chest and back with pruritus $2 \mathrm{~h}$ after taking $500 \mathrm{mg}$ of PV (1 \\
\hline 14 & 5 & $\mathrm{~F}$ & MPE & MPE on abdomen, legs, and chest after taking $200 \mathrm{mg}$ of cexime every \\
\hline 15 & 0.5 & M & MPE & Maculopapulae on abdomen and neck with facial erythema after $100 \mathrm{~m}$ \\
\hline 16 & 4 & $\mathrm{~F}$ & URT & Wheals with pruritus on chest, abdomen and legs after $250 \mathrm{mg}$ of AX e \\
\hline 17 & 3 & $\mathrm{~F}$ & URT & Generalized wheals with pruritus after taking $150 \mathrm{mg}$ of AX every $8 \mathrm{~h}$ \\
\hline 18 & 6 & M & URT & Pruritus followed by wheals on legs and buttocks, and facial erythema \\
\hline 19 & 8 & M & URT & Generalized pruritus with wheals on the back after taking $250 \mathrm{mg}$ of $\mathrm{A} Y$ \\
\hline 20 & 6 & $\mathrm{~F}$ & URT & Pruritus and wheals on legs and arms after taking $250 \mathrm{mg}$ of AX every \\
\hline 21 & 4 & $\mathrm{~F}$ & URT & Generalized wheals with pruritus after taking $250 \mathrm{mg}$ of AX every $8 \mathrm{~h}$ \\
\hline 22 & 1 & $\mathrm{~F}$ & URT & Wheals on abdomen, back, face and legs after taking $125 \mathrm{mg}$ of AX eve \\
\hline 23 & 4 & M & URT & Urticaria with pruritus on back that generalized after taking $250 \mathrm{mg}$ of \\
\hline 24 & 7 & $\mathrm{~F}$ & URT & Generalized wheals on abdomen, buttocks and back after taking 250 of \\
\hline 25 & 12 & $\mathrm{~F}$ & URT & Systemic pruritus with wheals on abdomen and legs $1.5 \mathrm{~h}$ minutes after \\
\hline 26 & 5 & M & URT & Skin pruritus followed by wheals on arms that extended to abdomen, ch \\
\hline 27 & 4 & $\mathrm{~F}$ & URT & Facial erythema and wheals on abdomen after taking $250 \mathrm{mg}$ of AX eve \\
\hline 28 & 1.5 & $\mathrm{M}$ & URT & Wheals on legs and neck, extended to chest $1.5 \mathrm{~h}$ after taking $125 \mathrm{mg}$ o \\
\hline 29 & 2 & $\mathrm{~F}$ & URT & Generalized wheals on chest, abdomen and legs after taking 100/12.5 \\
\hline 30 & 13 & $\mathrm{~F}$ & URT & Generalized pruritus with wheals on elbows, knees, and legs after takin \\
\hline 31 & 5 & $\mathrm{~F}$ & URT & Wheals on abdomen and neck, with joint pain after taking $250 \mathrm{mg}$ of \\
\hline 32 & 2 & $\mathrm{M}$ & $\mathrm{URT} / \mathrm{AE}$ & Facial and eyelid angioedema with wheals on back, abdomen and arms \\
\hline 33 & 3 & $\mathrm{~F}$ & $\mathrm{URT} / \mathrm{AE}$ & Generalized erythema and lips angioedema after taking 125 of AX every \\
\hline 34 & 11 & M & $\mathrm{URT} / \mathrm{AE}$ & Systemic pruritus with facial erythema, eyelid angioedema and wheals \\
\hline 35 & 4 & $\mathrm{M}$ & $\mathrm{URT} / \mathrm{AE}$ & Wheals on abdomen, face, and neck with eyelid angioedema after takin \\
\hline 36 & 15 & M & $\mathrm{URT} / \mathrm{AE}$ & Erythema and pruritus on face followed by wheals on arms and lips ang \\
\hline
\end{tabular}

F: female; M: male; PV: penicillin V; AX: amoxicillin; AX-CLAV: amoxicillin-clavulanic acid; MPE: maculopapular exanthema; URT: urticaria; URT/AE: urticaria/angioedema.

\section{FIGURE LEGENDS}

Figure 1. Allergological evaluation algorithm.

Figure 2. Time from drug provocation test (DPT) to reactions in children with nonimmediate drug hypersensitivity reactions. 

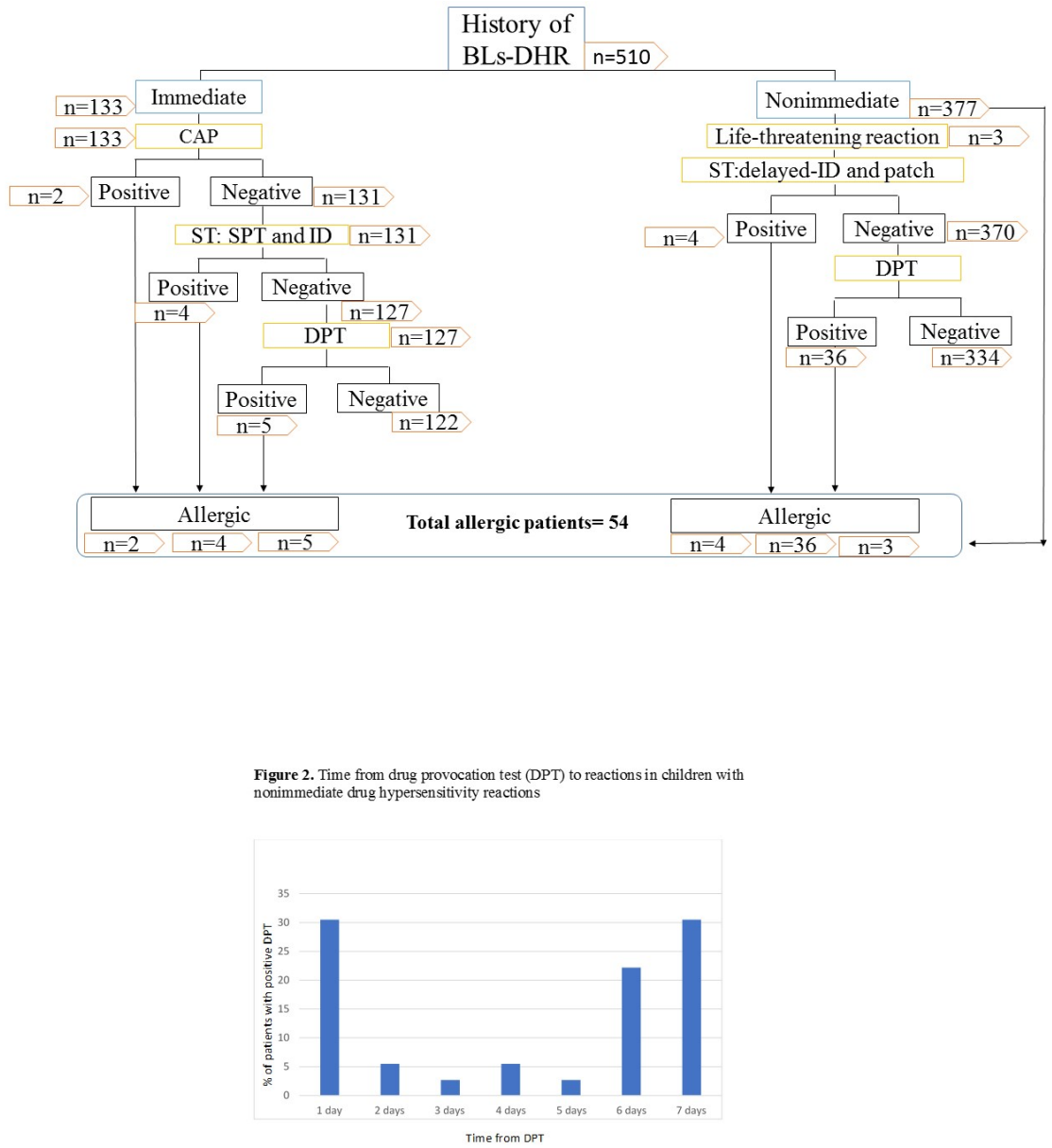